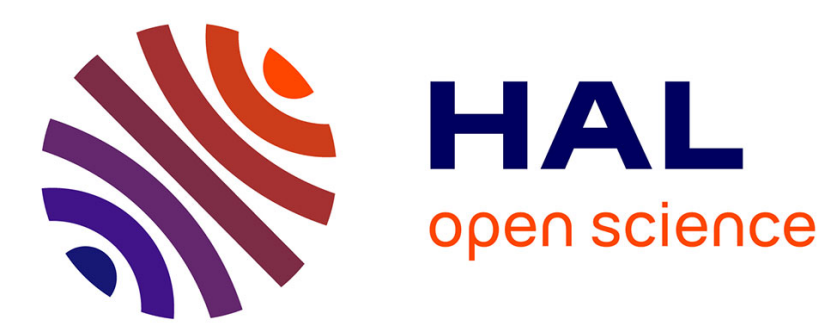

\title{
On optimal swinging of the biped arms
}

\author{
Yannick Aoustin, M. Formal'Skii
}

\section{To cite this version:}

Yannick Aoustin, M. Formal'Skii. On optimal swinging of the biped arms. 2008. hal-00462472

HAL Id: hal-00462472

https://hal.science/hal-00462472

Preprint submitted on 9 Mar 2010

HAL is a multi-disciplinary open access archive for the deposit and dissemination of scientific research documents, whether they are published or not. The documents may come from teaching and research institutions in France or abroad, or from public or private research centers.
L'archive ouverte pluridisciplinaire HAL, est destinée au dépôt et à la diffusion de documents scientifiques de niveau recherche, publiés ou non, émanant des établissements d'enseignement et de recherche français ou étrangers, des laboratoires publics ou privés. 


\title{
On optimal swinging of the biped arms
}

\author{
Y. Aoustin and A. M. Formal'skii
}

\begin{abstract}
A ballistic walking gait is designed for a planar biped with two identical two-link legs, a trunk and two onelink arms. This seven-link biped is controlled via impulsive torques at the instantaneous double support to obtain a cyclic gait. These impulsive torques are applied in six inter-link joints. Then infinity of solutions exists to find the impulsive torques. An energy cost functional of these impulsive torques is calculated to choose a unique solution by its minimization. Numerical results show that for a given time period of the walking gait step and a length of the step, there exists an optimal swinging amplitude of arms. For this optimal motion of the arms, mentioned above cost functional is minimum.
\end{abstract}

\section{INTRODUCTION}

Biped robots belong to the family of mechanical systems, which use the environment for their displacements. In consequence, their interaction with the ground is essential and many characteristics, problems have to be taken into account, underactuated [1], [2] or over actuated phases [16], impacts [9], [10], [14], balancing stability, [4], stability of cyclic motion, [1], [11], [12], [15]. In future many applications such as medical assistance, inspection or manipulation tasks in human environment would be entrusted to biped robots. Another role could be to understand some characteristics of the human walking. But it is difficult to use human as experimental testbed to understand the dynamics of the walk because the friction effects in joints are quasi null for human, the energetic cost of producing muscle forces is complex to analyze [8], the human walking gaits are the results of a complex orchestration of muscle forces, joint motions, and neural motor commands. However, from the mechanical point of view, the design of reference trajectories for bipeds, passive walking biped descending a gentle slope under gravity or ballistic walking bipeds can help to do some correlations with human experiments about the existence of a symmetry for the link motions, exchange of energy, displacement of the body mass center. If human and animal motions comprise alternating periods of muscle activity and relaxation, then it is logical to consider problem of purely ballistic swing phases and double support phases with impulsive inter-link torques. Similar statement of the problem is proposed in [9], [10], [17] and [15]. Perhaps this kind of motion with ballistic parts and impulsive torques appears to be less energy consuming, [10].

Chevallereau et al [6] verify that when the motion velocity of the legged robot becomes important it is cheaper to run

Y. Aoustin is with IRCCyN, UMR 6597, Ecole Centrale de Nantes, Université de Nantes, France (e-mail: Yannick.Aoustin@irccyn.ec-nantes.fr).

A. M. Formal'skii is with the Institute of Mechanics, Moscow Lomonosov State University, 1, Michurinskii Prospect, Moscow, 119192, Russia. (email: formal@imec.msu.ru) than to walk from the energy point of view. In [7], authors show that model results and experimental data both support the proposition that the primary function of the arms during gait is to reduce fluctuations in angular momentum. The orientation of the trunk, the varying length of the step can improve the stability of a cyclic walking gait [1], [2]. The ballistic running was considered by [18] with a study of symmetry properties of the joint variables and their velocities during the flight for a biped with massless feet.

We consider an anthropomorphic biped with one-link arms but without feet (with point feet). The physical parameters for a biped are chosen from average human data. The contribution of this paper is to solve a boundary value problem to find a walking ballistic gait with instantaneous double support and impulsive torques for this biped and to study the effect of arms. Furthermore we compute a minimum energy of the impulsive torques during the instantaneous double support phase. The research of these minimums is doing in function of the amplitude of the arms swinging. The impulsive control torques, which are applied in the inter-link joints between the neighboring single support phases are described by deltafunctions of Dirac. Of course it is not possible to realize these impulsive control torques. Therefore, our approach can be considered as asymptotic. Using this approach we can evaluate the role of the arms in the walking process.

Section II is devoted to the biped model presentation. Dynamic equations are presented, and the physical parameters of the model are chosen. Problem definition of the ballistic walking is given, algebraic equations for the instantaneous double support phase are designed, the methods to find the ballistic swing motion and to solve the algebraic equations are described in Section III. The calculation of the energy cost functional for the impulsive control is recalled in Section IV. Some interesting results, which are obtained from numerical investigations are presented in Section V. Our conclusion and perspectives are offered in Section VI.

\section{BIPED MODEL}

We describe here the equations of motion of the biped walking gait, which is composed of alternating single support and instantaneous double support phases.

\section{A. Equations of motion of the biped in single support phase}

The studied anthropomorphic biped, depicted in Figure 1 has a trunk, two identical one-link arms and two identical two-link legs. Its motion takes place in the sagittal plane. It has no feet. All bodies (links) are supposed massive and rigid and are connected by revolute joints. The two Cartesian coordinates $x, y$ of the hip joints and the seven 


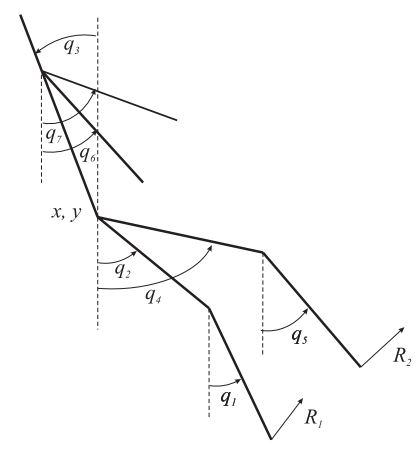

Fig. 1. Biped's diagram in the sagittal plane: Generalized Coordinates, Forces Applied to the Leg Tips.

orientation angles $q=\left(q_{1}, q_{2}, q_{2}, q_{3}, q_{4}, q_{5}, q_{6}, q_{7}\right)^{* 1}$ of the legs, torso and arms define vector $X$ of the nine generalized coordinates. The vector $\Gamma=\left(\Gamma_{1}, \Gamma_{2}, \Gamma_{3}, \Gamma_{4}, \Gamma_{5}, \Gamma_{6}\right)^{*}$ with six components denotes the applied torques in the hip joints, knee joints and shoulder joints. Let $R_{j}=\left(R_{j x}, R_{j y}\right)^{*}$ be the force applied to the point foot $j$. When leg $j$ is on the ground, then $R_{j}$ is the ground reaction. If leg $j$ is the swing leg, then $R_{j}=0$.

Using the second Lagrange method, the equations of motion of the planar seven-link biped in the swing phase are obtained and they have the following well-known matrix form, for supporting leg $j=1$ or 2 ,

$$
A(q) \ddot{X}+H(q, \dot{q})=D \Gamma+J_{j}^{*}(q) R_{j} .
$$

Here $A(q)$ is the symmetric, positive definite $9 \times 9$ inertia matrix; $H(q, \dot{q})$ is the $9 \times 1$ vector of the centrifugal, Coriolis and gravity forces; $D$ is a $9 \times 6$ fixed matrix, consisting of zeros and units; $J_{j}(q)$ is the $2 \times 9$ Jacobian matrix of constraint functions. These constraints are relative to the contact between the ground and the tip of the stance leg $j$. Setting null acceleration condition of the stance leg tip

$$
J_{j}(q) \ddot{X}+H_{j}(q, \dot{q})=0
$$

implies that both horizontal and vertical coordinates of that leg tip does not change, if its initial velocity is null. $H_{j}(q, \dot{q})$ is a $2 \times 1$ vector. Thus, in the single support the number of degrees of freedom is seven, but there are only six torques. This means that the biped is an under actuated mechanism during the single support phase.

\section{B. Double Support}

During the biped's gait, the impact occurs at the end of a single support phase, when the swing leg tip touches the ground. At the instant of impact, denoted by $T$, the double support phase is assumed instantaneous. We check this assumption a postiori in simulation. At the instant of the passive inelastic impact, the mechanism looses energy. Therefore, the velocity after the impact will not be the desired one, if the bearing surface is horizontal. Then for the next ballistic half step the desired initial velocity will

\footnotetext{
${ }^{1}$ Notation ${ }^{*}$ means transposition.
}

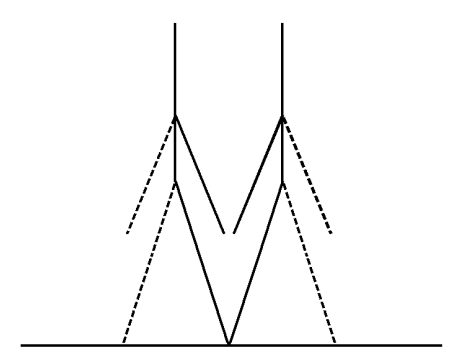

Fig. 2. Initial and final configurations of the biped.

not be reached. In consequence, a complete walking cyclic gait of the biped cannot be realized on a horizontal surface without active torques [11]. However, theoretically, around the instantaneous double support it is possible to define impulsive torques in order to ensure the desired velocity jump, see [13] and [6]. In Subsection III-B, it is shown how to define these impulsive torques.

\section{Physical parameters of the biped}

For the seven-link biped we use the physical parameters from [10]. The whole mass of the biped is $75 \mathrm{~kg}$, its height is $1.75 \mathrm{~m}$. The masses and the lengths of its seven links (Figure 1), are: $m_{1}=m_{5}=4.6 \mathrm{~kg}, m_{2}=m_{4}=8.6 \mathrm{~kg}$, $m_{3}=16.5 \mathrm{~kg}, l_{1}=l_{5}=0.497 \mathrm{~m}, l_{2}=l_{4}=0.41 \mathrm{~m}$, $l_{3}=0.625 \mathrm{~m}, l_{6}=l_{7}=0.66 \mathrm{~m}$.

The distance between the knee joint and the mass center of the shin is: $s_{1}=s_{5}=0.324 \mathrm{~m}$, between the hip joint and the mass center of thigh is: $s_{2}=s_{4}=0.18 \mathrm{~m}$, between the hip joint and the mass center of the trunk is: $s_{3}=0.386 \mathrm{~m}$ and between the shoulder joint and the mass center of the arm is: $s_{6}=s_{7}=0.33 \mathrm{~m}$. The distance between the shoulder joints and the hip joints is: $s_{b}=0.35 \mathrm{~m}$.

The inertia moments around the mass center of each link are: $B_{1}=B_{5}=0.0521 \mathrm{~kg} \cdot \mathrm{m}^{2}, B_{2}=B_{4}=B_{6}=B_{7}=$ $0.7414 \mathrm{~kg} \cdot \mathrm{m}^{2}, B_{3}=11.3 \mathrm{~kg} \cdot \mathrm{m}^{2}$.

\section{PRoblem DEFINITION OF BALLISTIC MOTION AND IMPULSIVE CONTROL}

\section{A. Single support}

Let $X(0)=[x(0), y(0), q(0)]$ be the initial configuration of the biped at time $t=0$. We assume the front and hind legs are a stance and swing legs correspondingly. The final configuration of the biped in the single support phase at a given time $t=T$ is noted $X(T)=[x(T), y(T), q(T)]$. This configuration is similar to the initial configuration with the swapping legs. In Figure 2, both boundary configurations $X(0)$ and $X(T)$ are shown. The left pose is the initial configuration, the right pose is the terminal configuration.

Let $L$ be the length of the half step corresponding to a single support. We consider a ballistic motion during the single support phase with $\Gamma=0$. Consequently the equation 


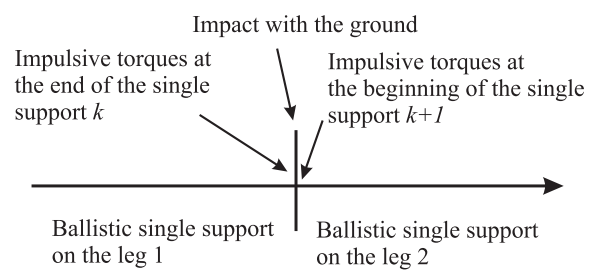

Fig. 3. Decomposition of the impulsive impact.

(1) for ballistic motion becomes,

$$
A(q) \ddot{X}+H(q, \dot{q})=J_{j}^{*}(q) R_{j} .
$$

To design the ballistic walking it is necessary to find the solution $X(t)=[x(t), y(t), q(t)]$ of system of equations (3) and (2) with the given boundary conditions $X(0)$ and $X(T)$. We have to find the initial velocity vector $\dot{X}(0)$ such that $X(t)$ reaches the given final configuration $X(T)$ at a given time $T$. This boundary value problem can be numerically solved using a Newton method with $\dot{X}(0)$ unknown. The motion of the biped is admissible, if the vertical component of the ground reaction is positive (is directed upwards) and if the swing leg moves over the ground as $0<t<T$. These constraints are checked after the boundary value problem solving.

After solving the boundary value problem the vector of initial velocities $\dot{X}(0)$ becomes known. We denote it by $\dot{X}^{a}$. If initial conditions $X(0), \dot{X}^{a}$ are known, then by integration of the system (3), (2) the vector of the terminal velocities $\dot{X}(T)$ can be found also. We denote it by $\dot{X}^{b}$.

\section{B. Double support}

Let us consider the current ballistic motion on the stance leg 1 and the following ballistic motion on the stance leg 2 . The final velocity vector $\dot{X}^{b}$ of the current ballistic swing motion and the initial velocity vector $\dot{X}^{a}$ of the next ballistic swing motion are known from the solution of the boundary value problem and the numerical integration of the equations of motion (3), (2). Let us apply the impulsive torques with the intensity vectors $I^{-}$and $I^{+}$respectively just before and just after the impact to create a complete cyclic motion. Then we divide the double support into three sub-phases and detail these sub-phases, which are presented in Figure 3.

- The swing leg 2 touches the ground at the end of the ballistic single support motion on the leg 1 , and an impact occurs. Just before the touching the ground, in the first sub-phase at time $T^{-}=T-0$, impulsive torques $\Gamma^{-}(t)=I^{-} \delta\left(t-T^{-}\right)$are applied at the interlink joints. At the same instant $T^{-}$the impulsive ground reaction $R_{1}^{-}=I_{R_{1}}^{-} \delta\left(t-T^{-}\right)$is applied in the hind leg tip. Here $\delta\left(t-T^{-}\right)$is the Dirac delta-function. $I_{R_{1}}^{-}\left(I_{R_{1 x}}^{-}, I_{R_{1 y}}^{-}\right)$is the vector of the magnitudes of the impulsive reaction in the leg 1 . Under the impulsive torques, the velocity vector $\dot{X}$ of the biped changes instantaneously from the value $\dot{X}^{b}$ to some value $\dot{X}^{-}$. The corresponding equations for the velocities jump can be obtained through integration of equations of motion
(1), (2) for the infinitesimal time from $T-0$ to $T$. The torques provided by the Coriolis and gravity forces have finite values. Thus, they do not influence the velocity jump:

$$
\begin{gathered}
A[q(T)]\left(\dot{X}^{-}-\dot{X}^{b}\right)=D I^{-}+J_{1}^{*}[q(T)] I_{R_{1}}^{-} \\
J_{1}[q(T)] \dot{X}^{-}=0_{2 \times 1}
\end{gathered}
$$

Here $q(T)$ denotes the configuration of the biped at the instant of impulsive actions (at the double support). This configuration does not change at the instants of the first, second and third sub-phases. The second equality (4) means that the velocity of the hind leg tip remains zero after the first sub-phase. The biped has the velocity vector $\dot{X}^{-}$just before the next sub-phase, which is passive impact.

- The second sub-phase is assumed to be a passive impact, i.e. without torques applied in the inter-link joints, absolutely inelastic and that the legs do not slip. Given these conditions, the ground reactions at the instant of an impact can be considered as impulsive forces and defined by the delta-functions $R_{j}=I_{R_{j}} \delta(t-T)$ $(j=1$, or 2$)$. Here $I_{R_{j}}\left(I_{R_{j x}}, I_{R_{j y}}\right)$ is the vector of the magnitudes of the impulsive reaction in the leg $j$, see [10]. The corresponding equations for the velocities jump can be obtained through integration of the matrix motion equation (1) for the infinitesimal time. The velocity of the stance leg tip 1 before an impact equals to zero (see the second equation in (4))

Generally speaking, two results are possible after the impact, if we assume that there is no slipping of the leg tips. The stance leg lifts off the ground or both legs remain on the ground. In the first case, the vertical component of the velocity of the taking-off leg tip just after the impact must be directed upwards. Also there is no interaction (no friction, no sticking) between the taking-off leg tip and the ground. The ground reaction in this taking-off leg tip must be null. In this case, the impact equations can be written in the following matrix form:

$$
A[q(T)]\left(\dot{X}^{+}-\dot{X}^{-}\right)=J_{2}^{*}[q(T)] I_{R_{2}}
$$

Here $\dot{X}^{+}$is the velocity vector just after an inelastic impact. The swing leg 2 after the impact becomes a stance leg. Therefore, its tip velocity becomes zero after the impact,

$$
J_{2}[q(T)] \dot{X}^{+}=0_{2 \times 1}
$$

In the second case, the both legs remain on the support after the passive impact. The ground produces impulsive reactions and their vertical components in both legs have to be directed upwards. But we consider the first case only. To do this we take into account the corresponding conditions: the vertical components of the velocity vector of the hind leg tip and of the ground reaction in the front leg have to be directed upwards. 
- The swing leg 1 takes off the ground at the second subphase, which is passive impact. After the next ballistic single support motion on the leg 2 starts. Just after the take off, in the third sub-phase at time $T^{+}=T+0$, impulsive torques $\Gamma^{+}(t)=I^{+} \delta\left(t-T^{+}\right)$are applied in the inter-link joints to change the velocity of the biped instantaneously from the velocity vector just after passive impact $\dot{X}^{+}$to the known velocity vector $\dot{X}^{a}$. Integrating the differential equations (1) we come to the following matrix relation:

$$
A[q(T)]\left(\dot{X}^{a}-\dot{X}^{+}\right)=D I^{+}+J_{2}^{*}[q(T)] I_{R_{2}}^{+}
$$

System (4)-(7) is composed of 31 scalar equations to find 36 unknown variables, which are the components of the vectors: $\dot{X}^{-}, I^{-}, I_{R_{2}}^{-}, \dot{X}^{+}, I_{R_{2}}, I^{+}$and $I_{R_{2}}^{+}$. Then infinity of solutions exists to solve this problem of impulsive control. But if the number of the equations is less than the number of unknown variables, it is possible to extract a unique solution minimizing some cost functional. The components of mentioned above vectors are subjects of minimization. Among this set of components five can be defined as parameters to minimize a cost functional. In the next Section IV, the chosen cost functional is presented. But solving system (4) - (7) it is necessary to take into account that the vertical component of the ground reactions can be never directed downwards. The vertical component of the hind leg tip velocity at the instant of take-off has to be directed upwards also.

\section{ENERGY COST OF IMPULSIVE CONTROL}

We assume that the actuators are not regenerative (energy cannot be restored in the drives). Then the motion energy cost functional is defined as in [5], [10] and [6]:

$$
W=\int_{T-0}^{T+0}\left|\Gamma^{*} \dot{\theta}\right| d t
$$

If the mentioned above assumption is relaxed, then the cost functional must be changed. But this is a problem to choose the corresponding functional.

In our case with impulsive torques, the cost functional (9) can be expressed as:

$$
W=\sum_{i=1}^{6}\left[\int_{T-0}^{T}\left|\Gamma_{i}^{-}(t) \dot{\theta}_{i}(t)\right| d t+\int_{T}^{T+0}\left|\Gamma_{i}^{+}(t) \dot{\theta}_{i}(t)\right| d t\right]
$$

where the joint variables $\theta_{i}$ are such as:

$$
\begin{aligned}
& \theta_{1}=q_{2}-q_{1}+\pi, \quad \theta_{2}=q_{3}-q_{2}+\pi, \\
& \theta_{3}=q_{3}-q_{4}+\pi, \quad \theta_{4}=q_{4}-q_{5}+\pi, \\
& \theta_{5}=q_{3}-q_{6}+\pi, \quad \theta_{6}=q_{3}-q_{7}+\pi .
\end{aligned}
$$

The calculation of the integrals in the expression (9) leads [10] to the following formulas:

$$
W=\sum_{i=1}^{6}\left(W_{i}^{-}+W_{i}^{+}\right)
$$

with

$$
\begin{gathered}
W_{i}^{-}=\left|I_{i}^{-} \frac{\dot{\theta}_{i}\left(T^{-}\right)+\dot{\theta}_{i}(T)}{2}\right| \text { if } \dot{\theta}_{i}\left(T^{-}\right) \dot{\theta}_{i}(T) \geq 0 \\
W_{i}^{-}=\left|I_{i}^{-} \frac{\dot{\theta}_{i}^{2}\left(T^{-}\right)+\dot{\theta}_{i}^{2}(T)}{2\left[\dot{\theta}_{i}\left(T^{-}\right)-\dot{\theta}_{i}(T)\right]}\right| \text { if } \dot{\theta}_{i}\left(T^{-}\right) \dot{\theta}_{i}(T)<0 \\
W_{i}^{+}=\left|I_{i}^{+} \frac{\dot{\theta}_{i}(T)+\dot{\theta}_{i}\left(T^{+}\right)}{2}\right| \text { if } \dot{\theta}_{i}(T) \dot{\theta}_{i}\left(T^{+}\right) \geq 0 \\
W_{i}^{+}=\left|I_{i}^{+} \frac{\dot{\theta}_{i}^{2}(T)+\dot{\theta}_{i}^{2}\left(T^{+}\right)}{2\left[\dot{\theta}_{i}(T)-\dot{\theta}_{i}\left(T^{+}\right)\right]}\right| \text {if } \dot{\theta}_{i}(T) \dot{\theta}_{i}\left(T^{+}\right)<0
\end{gathered}
$$

In simulation, with given length and time period of the step, for each arms amplitude, we choose a unique solution of the system (4) - (7) by minimizing the quantity (10).

\section{Simulations}

For several values of the length step and of the time period, we study here numerically the influence of the arm swinging on the energy cost of biped walking.

In Figure 4, the energy consumption as function of the amplitude of the arms swinging is shown for a fixed length of the half step $L=0.45 \mathrm{~m}$ and different time periods. All these curves correspond to the ballistic motions. In the boundary configurations of these motions, the legs are straight. The initial angle of the trunk inclination $q_{3}(0)=0^{\circ}$. The initial angles for the arm $q_{6}(0)$ belong to the interval $\left(0^{\circ}, 60^{\circ}\right)$ and $q_{7}(0)=-q_{6}(0)$.

By solid line, the curve with the time period $T=0.50 \mathrm{~s}$ and the half step length $L=0.45 \mathrm{~m}$ is drawn. We can see from this Figure 4 that with amplitude $2 \times 36.8^{\circ}$ the energy consumption is minimal. It means that this amplitude is optimal one for this case. In Figure 2, the boundary configurations namely for this case are shown.

By dashed line, the graph with less time period $T=0.45 \mathrm{~s}$ and the same half step length $L=0.45 \mathrm{~m}$ is drawn. We can see from this Figure 4 that the optimal amplitude becomes less. By dash-dot line, the graph with the larger time period $T=0.55 \mathrm{~s}$ and the same half step length $L=0.45 \mathrm{~m}$ is drawn. It follows from this Figure 4 that optimal amplitude becomes larger.

In Figure 5, the energy consumption as function of the amplitude of the arms swinging is shown for the fixed time period $T=0.50 \mathrm{~s}$ and a length half step $L$ varying $L=$ $0.40 \mathrm{~m}, L=0.45 \mathrm{~m}$ and $L=0.50 \mathrm{~m}$. If the length of the step increases, the optimal amplitude of the arms swinging decreases.

Thus, if the velocity of the biped walking decreases, the optimal amplitude of arms swinging increases.

These results complete the study of [7]. We can remark that for initial and final configurations, drawn in Figure 2 with a deviation of arms, close to the optimal deviation for a ballistic motion with the half step length $L=0.45 \mathrm{~m}$ and 


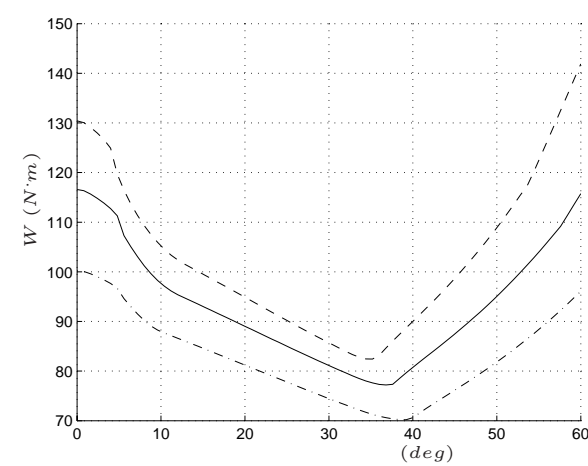

Fig. 4. For $L=0.45 m$, cost functional $W$ versus amplitude motion of the arms [degrees] $T=0.45 \mathrm{~s}$ (dashed), $T=0.50 \mathrm{~s}$ (solid), and $T=0.55 \mathrm{~s}$ (dashdot).

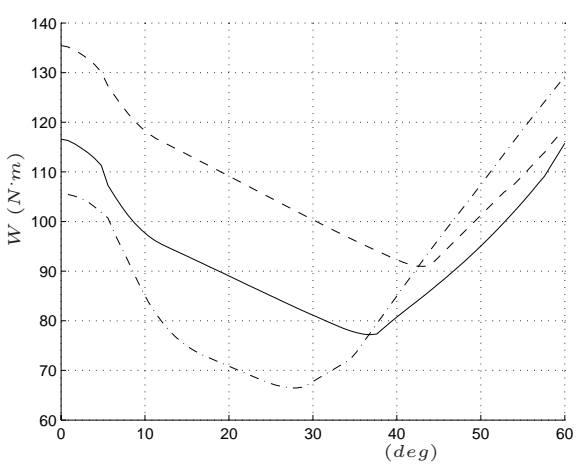

Fig. 5. For $T=0.50 \mathrm{~s}$, cost functional $W$ versus amplitude motion of the arms [degrees] $L=0.40 \mathrm{~m}$ (dashed), $L=0.45 \mathrm{~m}$ (solid), and $L=0.50 m$ (dashdot).

the time period $T=0.50 \mathrm{~s}$, the arms are almost parallel to the legs.

Figures 6-11, correspond to the ballistic motion with the time period $T=0.50 \mathrm{~s}$ and the half step length $L=0.45 \mathrm{~m}$ for the optimal amplitude of the arms swinging. In Figure 6, a plot of ballistic walking as a sequence of stick figures is shown. The swing leg moves over the support and bends with knee forward. The stance leg remains almost straight during the half step. These features haven't been prescribed in the statement of the problem previously. In the boundary configurations (see Figures 2,6), the legs are straight; the initial angle of the trunk inclination $q_{3}(0)=0^{\circ}$; the initial angles for the arms $q_{6}(0)=-q_{7}(0)=36.8^{\circ}$. Figures 7-9 show the behavior of the seven joint variables, Figures 10, 11 show the Cartesian position of the hip and the ground reaction components.

The absolute orientation of the stance tibia has a monotone evolution, which is well-known for a biped in its sagittal motion [2]. The trunk makes one vibration during one half step. Its motion is almost sinusoidal during the half step. The hip motion is similar to those of a human [3]. Figure 11 proves that the ballistic motion is valid because the vertical component of the ground reaction in the supporting leg is

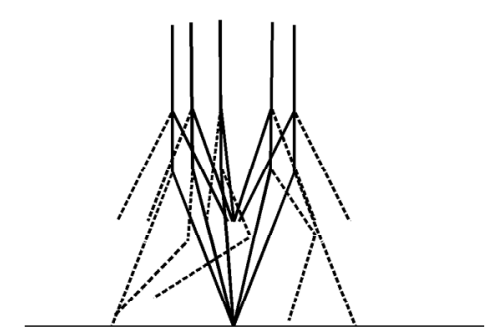

Fig. 6. Sequence of stick figures.
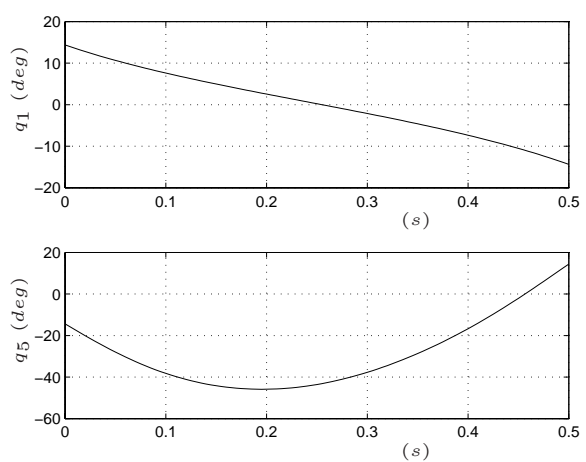

Fig. 7. Orientations [degrees] of tibias $q_{1}$ for the stance leg and $q_{5}$ for the swing leg versus time [seconds].

always positive.

The vertical displacement of center of mass (CoM) of the biped is less than $3.5 \mathrm{~cm}$ for all amplitudes of the arm swinging: from $0^{\circ}$ to $120^{\circ}$. The horizontal displacement of the CoM is not sensitive to the motion amplitudes of the arms.

Note, for the case $q_{3}(0)=0$ there are two kinds of boundary value problem solutions [10]. Following the initial velocity vector $\dot{X}(0)$ the solutions can lead to a symmetrical evolution of the variables relative to the time instant $T / 2$ or a non-symmetrical, which is the case here.
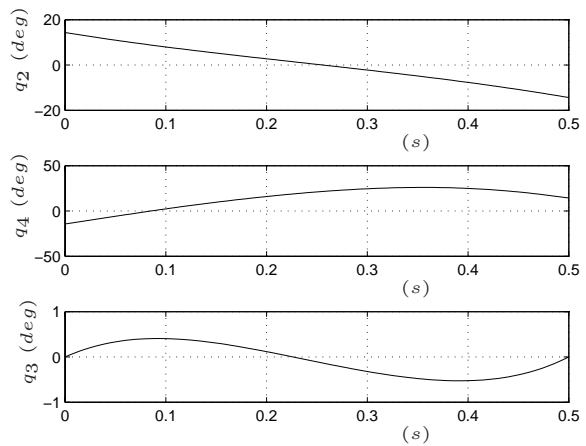

Fig. 8. Orientations [degrees] of the trunk $q_{3}$ and of femurs, $q_{2}$ for the stance leg and $q_{4}$ for the swing leg versus time [seconds]. 


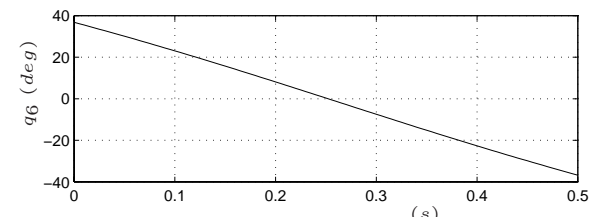

$(s)$

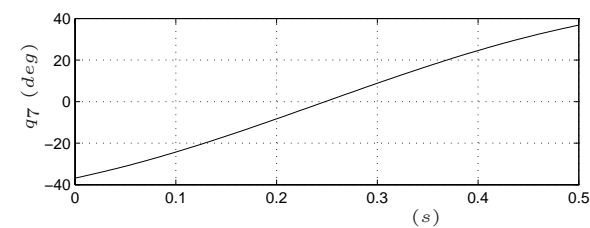

Fig. 9. Orientations [degrees] of arms, $q_{6}$ and $q_{7}$ versus time [seconds].
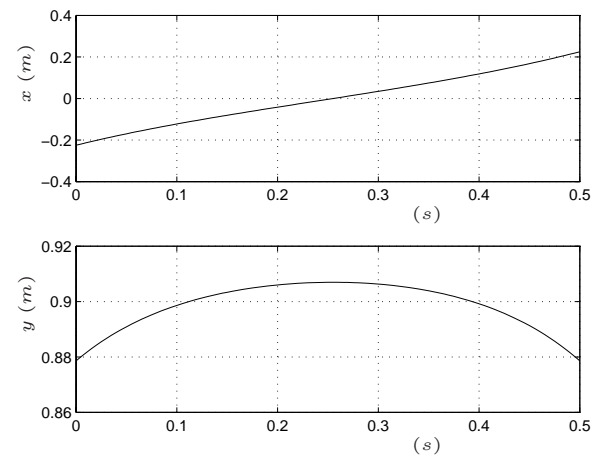

Fig. 10. Evolutions [meters] of Cartesian coordinates for the hip joints versus time [seconds].

\section{CONCLUSION}

Using ballistic trajectories and an impulsive control, numerically we have shown an existence of optimal amplitude of the arms swinging. The energy consumption for the walk is minimal, if the arms swing with this amplitude. If the velocity of the biped walking decreases, the optimal amplitude of arms swinging increases. The perspective is to extend the obtained results for a 3D biped model.

\section{REFERENCES}

[1] Y. Aoustin and A. M. Formal'skii. Design of reference trajectory to stabilize desired nominal cyclic gait of a biped. In Proc. of the International Workshop on Robot Motion and Control, ROMOCO'99, pages 159-165, 1999.

[2] Y. Aoustin and A.M. Formal'skii. Control design for a biped: reference trajectory based on driven angles as functions of the undriven angle. Int. J. of Computer and Systems Sciences, 42(4):159-176, 2003.

[3] H. Beaupied. Etude mécanique et énergétique de la marche, de la course et de la transition marche-course : influence de la spécialité athlétique. PhD thesis, Université de Rennes II France, 2003.

[4] C. Canudas-de Wit, B. Espiau, and C. Urrea. Orbital stabilization of underactuated mechanical system. In Proc. the World Congress IFAC (Cdrom), Barcelona, Spain, 2002.

[5] P. H. Channon, S. H. Hopkins, and D. T. Pham. Derivation of optimal walking motions for a bipedal walking robot. Robotica, 10(3):165172, 1992.

[6] C. Chevallereau, A.M. Formal'skii, and B. Perrin. Low energy cost reference trajectories for a biped robot. In Proc. of the IEEE Conf. on Robotics and Automation, volume 2, pages 1088-1094, 1998.
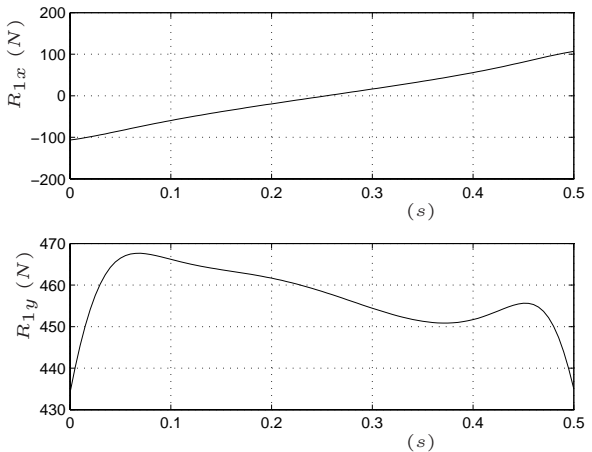

Fig. 11. Components of the ground reactions [Newton] versus time [seconds].

[7] S. Collins, P. Adamczyk, and A. Kuo. Swinging the arms makes walking easier. In Personnal communication from authors, 2007.

[8] J. Doke and A. D. Kuo. Energetic cost of producing cyclic muscle force, rather than work, to swing the human leg. The journal of experimental biology, 210:2390-2398, 2007.

[9] A. M. Formal'skii. Motion of anthropomorphic biped under implusive control. In Proc. of Institute of Mechanics, Moscow State Lomonosov University: "Some Questions of Robot's Mechanics and Biomechnics", pages 17-34, 1978, (In Russian).

[10] A.M. Formal'skii. Locomotion of Anthropomorphic Mechanisms. [In Russian], Nauka, Moscow, Russia, 1982.

[11] A. Goswami, B. Espiau, and A. Keramane. Limit cycles in a passive compass gait biped and passivity-mimicking control laws. Journal of Autonomous Robots, 4(3):273-286, 1997.

[12] J.W. Grizzle, G. Abba, and F. Plestan. Asymptotically stable walking for biped robots : analysis via systems with impulse effects. IEEE Transactions on Automatic Control, 46(1):51-64, 2001.

[13] Y. Hurmuzlu and T.-H. Chang. Rigid body collisions of a special class of planar kinematic chains. IEEE Transactions on systems, man and cybernetics, 22(5):964-971, 1992.

[14] Y. Hurmuzlu and D.B. Marghitu. Rigid body collisions of planar kinematic chains with multiple contact points. Int. Journal of Robotics Research, 13(1):82-92, 1994.

[15] T. McGeer. Passive dynamic walking. Int. J. of Robotics Research, 9(2):62-82, 1990.

[16] S. Miossec and Y. Aoustin. A simplified stability study for a biped walk with underactuated and overactuated phases. Int. Journal of Robotic Research, 24(6):537-551, June 2005.

[17] S. Mochon and T.A. McMahon. Ballistic walking: An improved model. Mathematical Bio-sciences, 52:241-260, 1981.

[18] D. D. Rogozhin. Impulsive control for anthropomorphic mechanism in the running with instantaneous support phase. Theory and Practice in Physical Training (In Russian), (5):27-38, 1989. 